\title{
Impacts of trait anxiety on visual working memory, as a function of task demand and situational stress.
}

David M. Spalding ${ }^{1}$, Marc Obonsawin, Caitie Eynon, Andrew Glass, Lindsay Holton, Monica McGibbon, Calhoun L. McMorrow, and Louise A. Brown Nicholls².

School of Psychological Sciences \& Health, University of Strathclyde, 40 George Street, Glasgow, G1 1QE.

1. ORCID ID: 0000-0002-4091-8823

2. Corresponding Author. Email: 1.nicholls@strath.ac.uk; telephone +44 (0)141 5482661. ORCID ID: 0000-0003-3520-6175; Twitter: @LNichollsStrath.

This manuscript version is the authors' accepted manuscript (after peer review).

To cite: Spalding, D. M., Obonsawin, M., Eynon, C., Glass, A., Holton, L., McGibbon, M.,

McMorrow, C. L., \& Nicholls, L. A. B. (in press). Impacts of trait anxiety on visual working memory, as a function of task demand and situational stress. Cognition \& Emotion. 


\begin{abstract}
Higher trait anxiety can impair cognitive functioning via attention, but relatively little is known about the impacts on visual working memory. These were investigated using previously validated visual feature binding tasks. In Study 1, participants' memory for visual features (shapes) and feature bindings (coloured shapes) was assessed. Stimulus presentation was simultaneous or sequential, varying attentional demand, and participants were grouped according to trait cognitive anxiety (low, moderate, high). No reliable effect of trait anxiety, either cognitive or somatic, was found on memory accuracy, but moderate trait cognitive anxiety was associated with faster correct response times (i.e. increased efficiency) when stimuli were sequentially presented. In Study 2, the role of situational stress was explored during a simultaneously presented task. Higher trait cognitive and somatic anxiety were both associated with poorer efficiency during both shape and binding memory tasks. Trait somatic anxiety also predicted poorer binding effectiveness (i.e. accuracy), in those reporting higher state cognitive anxiety. Situational stress predicted binding effectiveness, but never interacted with trait anxiety, and was therefore not necessary to observe these trait anxiety-visual working memory relationships. Trait cognitive and somatic anxiety, and situational stress, therefore each influence visual working memory performance.
\end{abstract}

Keywords: visual short-term/working memory; visual feature binding; executive function; trait anxiety; situational stress. 


\section{Impacts of trait anxiety on visual working memory, as a function of task demand and situational stress}

Attentional Control Theory (ACT; Eysenck, Derakshan, Santos, \& Calvo, 2007) predicts that anxiety can exert negative effects on cognitive performance via attention. Higher levels of anxiety (primarily trait) tend to reduce available executive resources, while simultaneously increasing the automatic capture of attention at the perceptual level. However, the predictions of ACT require more scrutiny, specifically regarding the effects of anxiety on visual working memory (Baddeley, Banse, Huang, \& Page, 2012; Moran, 2016), and in the context of current visual working memory paradigms.

\section{Visual working memory and attention}

Visual working memory is responsible for moment-to-moment processing and retention of visual information (Baddeley, 2012; Cowan, 2010; Logie, 2011; Luck, \& Vogel, 1997, 2013; Ma, Husain, \& Bays, 2014). It is generally agreed that successful temporary visual storage is dependent on attentional processes. Encoding into, and maintaining visual information within, visual working memory requires sustained attention (Chun, 2011), including the ability to inhibit internal cognitive distraction and external perceptual distractors (Bleckley, Foster, \& Engle, 2015; Chun, Golomb, \& Turke-Browne, 2011). Sustained attention to information, in the face of internal and external distraction, can be described as the focus of attention (Cowan, 2010). Allen, Baddeley, and Hitch (2017) highlighted the dissociation between top-down, executive-driven attention that can be used to achieve specific task goals (e.g. prioritising particular items of information) and the relatively automatic, or 'cost-free' nature of stimulus-driven attention that 
can result in enhanced memory for the most recently presented items. These attentional processes each allow stimuli to be more directly accessible to conscious awareness in working memory, with enhanced recall (Allen, Baddeley, \& Hitch, 2014; Hu, Hitch, Baddeley, Zhang, \& Allen, 2014; Hu, Allen, Baddeley, \& Hitch, 2016). However, representations enhanced by goal- or stimulus-driven attention are relatively vulnerable to being overwritten by subsequent stimuli.

Feature binding is the process by which multiple features (e.g. shape, colour, location) are encoded, maintained, and recalled as integrated objects (Allen, Baddeley, \& Hitch, 2006; Luck \& Vogel, 1997; Wheeler \& Treisman, 2002). Memory for the associations (typically in comparison to memory for individual features) may be assessed via recognition (change detection) or cued recall, and memoranda are typically presented in simultaneous or sequential arrays of 3-5 objects (Cowan, 2010). Memory for simultaneously presented individual features and multi-feature conjunctions is disrupted to a similar extent by executive load (Allen et al., 2006; Allen, Hitch, Mate, \& Baddeley, 2012; but see Brown \& Brockmole, 2010; Fougnie \& Marois, 2009). However, when objects are presented sequentially, binding memory is reliably reduced relative to individual feature memory (Allen et al. 2006; Baddeley, Allen, \& Hitch, 2011; Brown \& Brockmole, 2010; Gorgoraptis, Catalao, Bays, \& Husain, 2011). Specifically, memory for objects presented earlier in a sequence is typically poorer than for recently presented objects, especially the final one (Hu et al., 2014), suggesting that bound representations are particularly fragile. Sequential binding tasks have also illuminated the relationships amongst executive attention, perceptual attention, and working memory by varying task demands. Increased executive load reduces memory for sequentially presented objects other than the final item, and to a greater degree for binding than individual feature memory (Allen et al., 2014). These results 
suggest that executive resources are especially required to maintain earlier items in visual working memory.

\section{Anxiety, attention, and working memory}

Anxiety is a multi-dimensional construct that varies in intensity between individuals and situations. State anxiety refers to aroused anxious responses to specific stressors, while trait anxiety refers to an ongoing propensity for an individual to experience anxious states, akin to enduring worry or apprehension (Moran, 2016). Anxiety has cognitive (e.g. uncomfortable thoughts, poor concentration) and somatic (e.g., rapid heartbeat, fast and shallow breathing) dimensions, and these can be measured at both the trait and state level (Grös, Antony, Simms, \& McCabe, 2007; Ree, French, MacLeod, \& Locke, 2008). The relationship between anxiety, attention, and memory performance is based primarily on evidence from studies measuring trait anxiety (Eysenck et al., 2007; Eysenck \& Derakshan, 2011), with evidence indicating negative effects on executive attention in tasks involving both threatening and neutral stimuli (Berggren \& Derakshan, 2013).

One assumption of ACT (Eysenck et al., 2007) is that anxiety impairs attentional control due to the demand on executive resources. A second assumption is that this is compounded by hypervigilance to external stimuli, resulting in more perceptual stimuli to process, and restricted resources with which to process them. In support of this claim, individuals with high trait anxiety show increased response time (RT) in the presence of an irrelevant distractor (Moser, Becker, \& Moran, 2012), and RT increases as perceptual load increases (Sadeh \& Bredemeier, 2011). This demonstrates an association between anxiety and impaired stimulus filtering, and supports the 
view that executive resources, responsible for filtering, are reduced with anxiety. Participants' performance may therefore be slower, but not necessarily less accurate. However, the effect may also partly be the result of additional stimuli occupying storage in working memory, or reduced working memory capacity more generally (Moran, 2016).

\section{Anxiety and visual cognition}

Regarding the potential impacts of anxiety on visual cognition, in the long-term memory context, two recent studies demonstrated a tendency for participants to remember features of visual objects differently, depending on induced positive or negative affect (Spachtholz \& Kuhbandner, 2017; Spachtholz, Kuhbandner, \& Pekrun, 2016). However, there has been limited study of the impact of trait anxiety on visual working memory, particularly with respect to specific working memory mechanisms.

Previous evidence indicated that trait anxiety impacts the central executive component of working memory, but not the phonological or visuospatial processing and storage components (Christopher \& MacDonald, 2005; Eysenck, Payne, \& Derakshan, 2005). However, both storage capacity and stimulus filtering in visuo-spatial working memory may indeed be negatively affected by anxiety. Yao, Chen, and Qian (2018) found that trait anxiety predicted reduced storage capacity for faces. However, highly trait anxious individuals may not necessarily exhibit behavioural deficits during task performance, while distinct patterns of neural activity are still observable. These have been argued to reflect reduced visuo-spatial working memory storage capacity (Qi, Chen, et al., 2014), and inefficient filtering of distractors specifically during working memory maintenance (Qi, Ding \& Li, 2014; see also Bishop, 2007, for a review). 
Individuals with higher trait anxiety have even been shown to be faster and better able to identify whether an additional to-be-remembered stimulus was presented during a visual search task (Berggren, Blonievsky, \& Derakshan, 2015). However, such enhanced detection at the early stages of a visual working memory task could negatively impact performance at other stages, such as storage and recall.

Other researchers have found effects of state anxiety on working memory. Berggren, Curtis, and Derakshan (2017) showed that higher self-reported state anxiety was associated with poorer memory for orientations, when preceded by a neutral face. Furthermore, external threat (fearful cues) was associated with poorer filtering of distractors in those with higher state anxiety. With respect to mood inductions, anxiety induced via threat-of-shock has been associated with poorer visuo-spatial working memory performance (Shackman et al., 2006; Vytal, Cornwell, Letkiewicz, Arkin \& Grillon, 2013). Also, during an induced unpleasant affective state, higher trait anxiety limited neural activity indicative of task-relevant storage capacity in visual working memory (Figueira et al., 2017), but there were no observable effects on behavioural outcomes in this study. Thus, storage capacity and/or executive resources may be involved in the relationship between anxiety and visual working memory, but the precise factors giving rise to behavioural deficits requires further investigation.

To our knowledge, the impacts of anxiety have yet to be investigated fully in the context of a visual binding paradigm, and with manipulations such as sequential presentation, which could influence findings due to the additional demand imposed. Furthermore, to our knowledge, the influence of affect induction procedures on visual binding has not yet been investigated. We are aware of one study that investigated the impact of worry on visual feature binding in working 
memory. Moreno, Ávila-Souza, Gomes, and Gauer (2015) compared high and low worriers in a simultaneously presented visual binding task in which participants had to indicate whether an item had appeared in an initial array. High worriers were generally slower to respond (i.e., to both individual and bound features), and binding memory was generally less accurate than individual feature memory (i.e., for both worry groups), but no interactions were reported. Interestingly, Jaiswal, Tsai, Juan, Liang and Muggleton (2018) found reduced change detection ability for arrays of coloured squares (requiring shape-location binding) in those with higher levels of trait anxiety, as well as lower levels of mindfulness. However, binding performance was not directly compared with individual feature memory in this study, therefore the specificity of this effect remains unclear. More research using a visual binding paradigm is therefore clearly required, particularly using more comprehensive measures of the different dimensions of anxiety and including relevant task and situational manipulations. The present research was therefore designed to assess visual feature binding as a function of trait anxiety, attentional demand (Study 1), and situational stress (Study 2).

\section{Study 1}

We first assessed whether trait anxiety was associated with impairments in working memory for visual features and/or bindings, and the potential role of the attentional demand of the task. Binding-specific deficits are generally evident when memory arrays are presented sequentially, rather than simultaneously (Allen et al., 2006; Brown \& Brockmole, 2010; Brown, Niven, Logie, Rhodes, \& Allen, 2017), as bound representations are susceptible to overwriting (Hu et al., 2014). Therefore, in individuals reporting varying levels of trait anxiety, it is of 
interest to examine individual shape versus binding (coloured shape) memory performance under simultaneous and sequential presentation conditions. We predicted a three-way interaction between trait cognitive anxiety (low, moderate, or high), memory type (shape or binding), and presentation format (simultaneous or sequential). According to ACT (Eysenck et al., 2007), individuals high in trait anxiety tend to have reduced central executive resources available to deal with increased perceptual demands (Eysenck \& Derakshan, 2011; Pacheco-Unguetti, Acosta, Callejas, \& Lupiáñez, 2010). Because cognitive anxiety has been shown to affect central executive processes while somatic symptoms do not (e.g. Edwards, Edwards, \& Lyvers, 2015, 2017), the cognitive component of anxiety was of primary interest in the present study. It was therefore expected that participants with high trait cognitive anxiety would exhibit poorer binding memory relative to shape memory performance (i.e. a binding deficit), specifically when stimuli were presented sequentially. However, in order to assess the specificity of any effect of anxiety, we also analysed the potential influence of trait somatic anxiety. Additionally, regarding the sequential task, focused analyses could potentially reveal that those with higher anxiety exhibit greater binding deficits at certain (e.g. earlier) serial positions, which require more executive resources (Allen et al., 2006; Hu et al., 2014; Hu et al., 2016), so serial position was also investigated.

Method

\section{Participants}

Ninety-one participants (65 females) aged 17-27 years $(M=20.69, S D=1.86)$ were recruited from an undergraduate participant pool at the University of Strathclyde (gaining course credit for 
their participation), and via opportunity sampling. With the aim of dividing the sample into three anxiety groups, this sample size surpassed the sample sizes that have previously shown interactions between the task variables presently used (e.g. Brown et al., 2017). All participants reported normal or corrected-to-normal vision, and no memory impairments.

\section{Design}

A 3 (trait cognitive anxiety group: low/moderate/high, between participants) x 2 (memory type: shape/binding, within participants) x 2 (presentation type: simultaneous/sequential, within participants) mixed factorial design was used. Participants were separated into the three anxiety groups via a tertiary split of trait cognitive anxiety scores (see Table 1). The dependent variables were recall accuracy (proportion correct), as a measure of effectiveness in the task, and RT during correct trials (ms), as a measure of efficiency.

Table 1. Study 1 participant demographics, by trait cognitive and somatic anxiety group.

\begin{tabular}{llcccc}
\hline & N & $\begin{array}{c}\text { Anxiety**} \\
\text { Min-Max, } M(S D)\end{array}$ & $\begin{array}{c}\text { Age } \\
M(S D)\end{array}$ & $\begin{array}{c}\text { Sex } \\
(\mathrm{M}: \mathrm{F})\end{array}$ & $\begin{array}{c}\text { Yrs Education } \\
M(S D)\end{array}$ \\
\hline Trait Cognitive & 91 & & & \\
\hline Low & 32 & $11-19,15.38(2.50)$ & $20.81(2.43)$ & $13: 19$ & $15.72(1.61)$ \\
Moderate & 32 & $20-25,22.31(1.80)$ & $20.22(1.41)$ & $9: 23$ & $15.63(1.60)$ \\
High & 27 & $26-40,30.44(3.65)$ & $21.11(1.45)$ & $4: 23$ & $15.96(1.26)$ \\
\hline Trait Somatic & $90^{*}$ & & & & \\
\hline Low & 25 & $11-14,12.48(1.23)$ & $21.56(2.31)$ & $10: 15$ & $16.24(0.97)$ \\
Moderate & 31 & $15-18,16.35(1.20)$ & $20.13(1.43)$ & $8: 23$ & $15.55(1.75)$ \\
High & 34 & $19-44,24.41(5.71)$ & $20.44(1.48)$ & $8: 26$ & $15.62(1.56)$
\end{tabular}

${ }^{*}$ One participant excluded due to missing trait somatic anxiety data. ${ }^{* *}$ Reported anxiety measure within same dimension as grouping. 


\section{Materials}

Anxiety measure: The State-Trait Inventory for Cognitive and Somatic Anxiety (STICSA; Ree, et al., 2008) consists of two, self-report measures of cognitive (10 items) and somatic (11 items) anxiety. Cognitive items include "I can't get some thought out of my mind" and "I cannot concentrate without irrelevant thoughts intruding". Somatic items include "my heart beats fast" and "my breathing is fast and shallow". The STICSA is highly reliable, with excellent validity in control and patient populations, and has been argued to provide a more direct measure of anxiety that is correlated less with measures of depression (Grös et al., 2007; Ree et al., 2008) than the more commonly employed State-Trait Anxiety Inventory (STAI; Spielberger, Gorsuch, Lushene, Vagg, \& Jacobs 1983). In the STICSA-Trait questionnaire, participants indicate, on a 4-point Likert scale, how often, in general, each statement is true of them $(1=$ almost never, to $4=$ almost always). In the STICSA-State questionnaire, participants indicate how they feel right now, at this very moment, even if this is not how they usually feel $(1=$ not at all, to $4=$ very much so). Higher scores indicate higher anxiety. Both trait and state cognitive scores range from 10 to 40 , while trait and state somatic scores range from 11 to 44 .

Memory task: A single-probe change detection paradigm was used, administered via EPrime 2.0 software (Psychology Software Tools; Figure 1; e.g. Allen et al., 2006; Brown et al., 2017). In each trial, the memory array comprised three coloured shapes presented against a grey background. Objects consisted of one of six colours (blue, cyan, green, purple, red, \& yellow) and one of six shapes (arrow, circle, diamond, heart, plus, \& triangle). Test probes were a single blank (grey) shape with a black outline (in shape memory trials), or a coloured shape (for binding trials). For the shape memory task, 'lure' trials involved shapes that had not been 
presented within the array whereas, in the binding task, lure trials involved feature swaps between objects presented in the array, such that performance depended on retaining the specific combinations. Stimuli measured approximately $2 \mathrm{~cm}^{2}$ on screen. Viewing distance was not constrained.

\section{Procedure}

Ethical approval was obtained from the School of Psychological Sciences \& Health Ethics Committee at the University of Strathclyde, prior to data collection. After providing informed consent, all participants first completed the STICSA-Trait, then STICSA-State. There were four blocks of trials (shape and binding memory, each with simultaneous and sequential presentation). Administration order was counterbalanced, but both memory conditions were always performed within a particular presentation format before moving on to the subsequent presentation format, and the memory conditions were always conducted in the same order across presentation format conditions. Each trial block included six practice trials followed by 36 experimental trials, the order of which was randomised for each participant.

Participants began each trial by pressing the spacebar (see Figure 1). Participants were then shown a random two-digit number (between 20 and 99) for $2000 \mathrm{~ms}$, which they were asked to repeat aloud consistently, at a pace of approximately two repetitions per second, from initial number presentation until making a key-press response at the end of the trial. This was to 
minimise verbal encoding of stimuli (Baddeley, 2007; see also Brown et al., 2017) ${ }^{1}$. A fixation cross then appeared in the centre of the screen for the duration of the trial. After $1000 \mathrm{~ms}$ this was accompanied by the memory array, which was displayed above fixation. Simultaneous arrays were displayed for $750 \mathrm{~ms}$, while objects in the sequential arrays were presented for 250 $\mathrm{ms}$ each, one after the other from left to right. Following a $1000 \mathrm{~ms}$ delay, the test probe was presented below fixation at a randomised location (left, right, or centre) until participants made a key-press response. Participants indicated whether the item had been present in ( $z$ key) or absent from ( $m$ key) the memory array. The test item had appeared in the memory array in half of the trials in each block.

\footnotetext{
${ }^{1}$ A 3 (trait cognitive anxiety; low, moderate, high) x 2 (presentation format; simultaneous, sequential) x 2 (memory type; shape, binding) mixed analysis of variance (ANOVA) indicated consistency in articulation rate across conditions (all $p>.08)$. The grand mean number of articulations per trial was $6.21(S D=$ $1.21)$.
} 
Figure 1. Two example trials from Study 1 paradigm, for simultaneous (top) and sequential (bottom) presentation conditions. Stimuli are not drawn to scale, and different colours are represented by different fill effects. Trials were blocked by shape or object (binding) memory test probes.

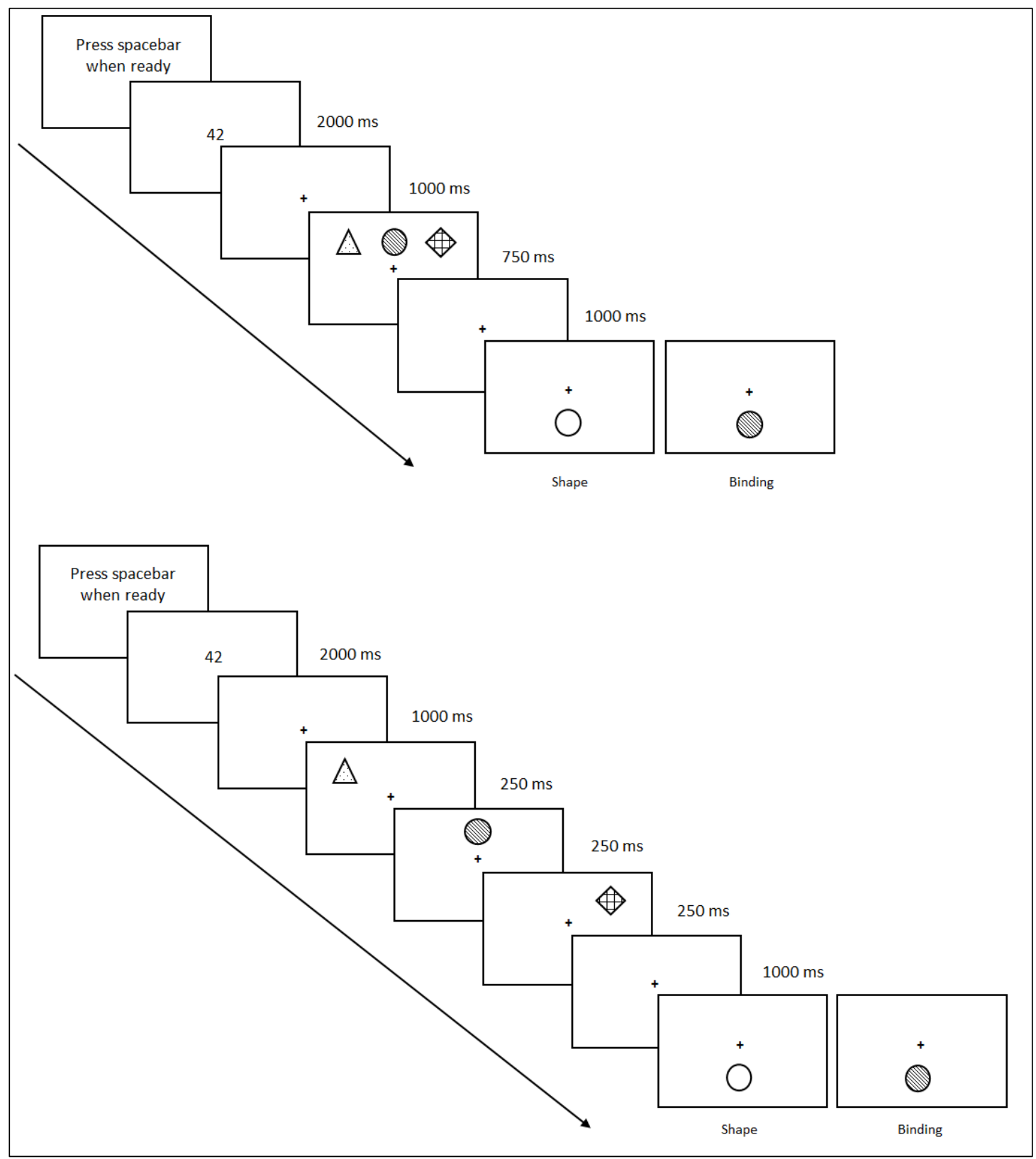




\section{Results}

The accuracy data (see Figure 2), and RT data from correct trials only (see Table 2), were analysed using 3 (trait cognitive anxiety; low, moderate, high) x 2 (presentation format; simultaneous, sequential) x 2 (memory type; shape, binding) mixed factorial analyses of variance (ANOVAs). Note, any follow-up t-tests used Bonferroni correction.

For accuracy, there were main effects of presentation format, $F(1,88)=20.13, M S E=$ $.007, p<.001, \eta_{p}^{2}=.19$, and memory type, $F(1,88)=9.61, M S E=.007, p=.003, \eta_{p}^{2}=.10$. Furthermore, as expected, there was a two-way interaction between presentation and memory type, $F(1,88)=3.98, M S E=.005, p=.049, \eta_{p}^{2}=.04$. Paired t-tests indicated that, overall, in the sequential presentation condition, shape memory $(M=.76, S D=.10)$ was better than binding memory $(M=.72, S D=.12 ; t(90)=3.72, p<.001)$, while there was no significant difference between these in the simultaneous condition $\left(M \operatorname{SHAPE}=.78, S D=.10 ; M_{\text {BINDING }}=.77, S D=.11\right.$; $p=.26)$. Regarding trait cognitive anxiety, it is notable that performance appears to be reduced with each increasing anxiety level specifically in the most demanding version of the task, that is, for binding memory under sequential presentation conditions (Figure 2). There were however no significant effects or interactions involving trait cognitive anxiety (all $p>.24){ }^{2}$ This was also the case when separately analysing hit and false alarm rates (all $p>.20)$.

\footnotetext{
${ }^{2}$ Multilevel modelling was also used, including trait cognitive anxiety as a continuous variable. This showed that performance was significantly predicted by presentation $(F=20.60, p<.001)$ and memory type $(F=9.58, p=.003)$, in line with the mixed ANOVA. The interaction between presentation format and memory type was not significant, though $(F=3.55, p=.063)$. Again, there were no significant effects involving trait cognitive anxiety (all $p>.13$ ).
} 
Figure 2. Performance accuracy (proportion correct, $\pm S E$ ) in Study 1, as a function of trait cognitive anxiety group, presentation format, and memory type.



Regarding RT during correct trials (Table 2), there was a two-way interaction between trait cognitive anxiety and presentation format, $F(2,88)=4.76, M S E=23560, p=.011, \eta_{p}^{2}=.10$

(Figure 3; all other $p>.25) .^{3}$ Paired-samples t-tests indicated that, in the moderate anxiety group, correct RT was significantly faster with sequential $(M=871, S D=189)$ than simultaneous $(M=936, S D=250)$ presentation, $t(31)=2.91, p=.007$. There was no reliable difference within the low $(p=.49)$ or high $(p=.15)$ anxiety groups. ${ }^{4}$

\footnotetext{
${ }^{3}$ Multilevel modelling indicated no main effects or interactions relating to correct RT data (all $p>.41$ ).

${ }^{4}$ When efficiency was operationalised as (accuracy/correct RT) x 1000 (in line with Edwards et al., 2015, 2016), there were no significant effects involving trait cognitive anxiety group (all $p>.25$ ).
} 
Table 2. Mean response times (ms) during correct trials (with $S D$ s) during shape and binding memory performance, under simultaneous and sequential presentation conditions for each trait cognitive anxiety group in Study 1.

\begin{tabular}{lcccc}
\hline & \multicolumn{2}{c}{ Simultaneous } & \multicolumn{2}{c}{ Sequential } \\
& Shape & Binding & Shape & Binding \\
\hline Low & 966 & 964 & 975 & 989 \\
Moderate & $(225)$ & $(224)$ & $(265)$ & $(305)$ \\
& 942 & 929 & 879 & 863 \\
High & $(233)$ & $(284)$ & $(226)$ & $(172)$ \\
& 973 & 984 & 1044 & 1024 \\
& $(278)$ & $(259)$ & $(377)$ & $(339)$ \\
\hline
\end{tabular}

Figure 3. Performance efficiency (correct response time, $\pm S E$ ) in Study 1, as a function of trait cognitive anxiety group and presentation format.

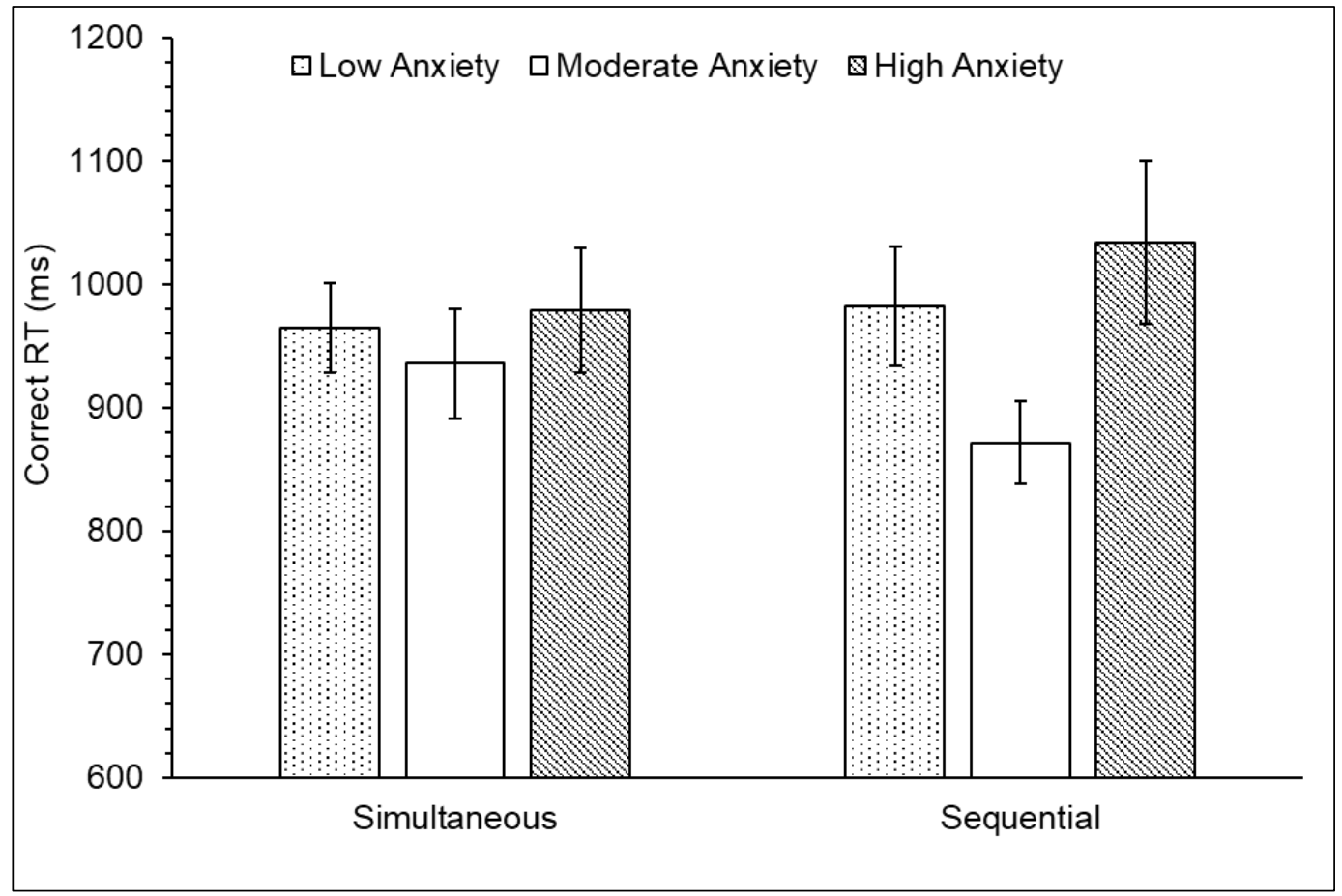




\section{Serial position}

To determine whether performance accuracy in the sequential task was influenced by serial position in the array, a 3 (trait cognitive anxiety group) x 2 (memory type) 3 (serial position; 1,2 , 3) mixed ANOVA was conducted (see Figure 4). In addition to a significant main effect of memory type as specified above $(p=.010)$, there was a main effect of serial position, $F(2,176)=$ $5.49, M S E=.050 p=.005, \eta_{p}^{2}=.06$. Performance at the middle position $(M=.69, S D=.22)$ was significantly poorer than at the first $(M=.75, S D=.18 ; p=.010)$ and last $(M=.76, S D=.18 ; p$ $=.012)$ positions. There were no other significant effects (all $p>.33)$. 
Figure 4. Performance effectiveness (proportion correct responses, $\pm S E$ ) in the sequential presentation condition of Study 1 , as a function of trait cognitive anxiety group, memory type, and serial position.

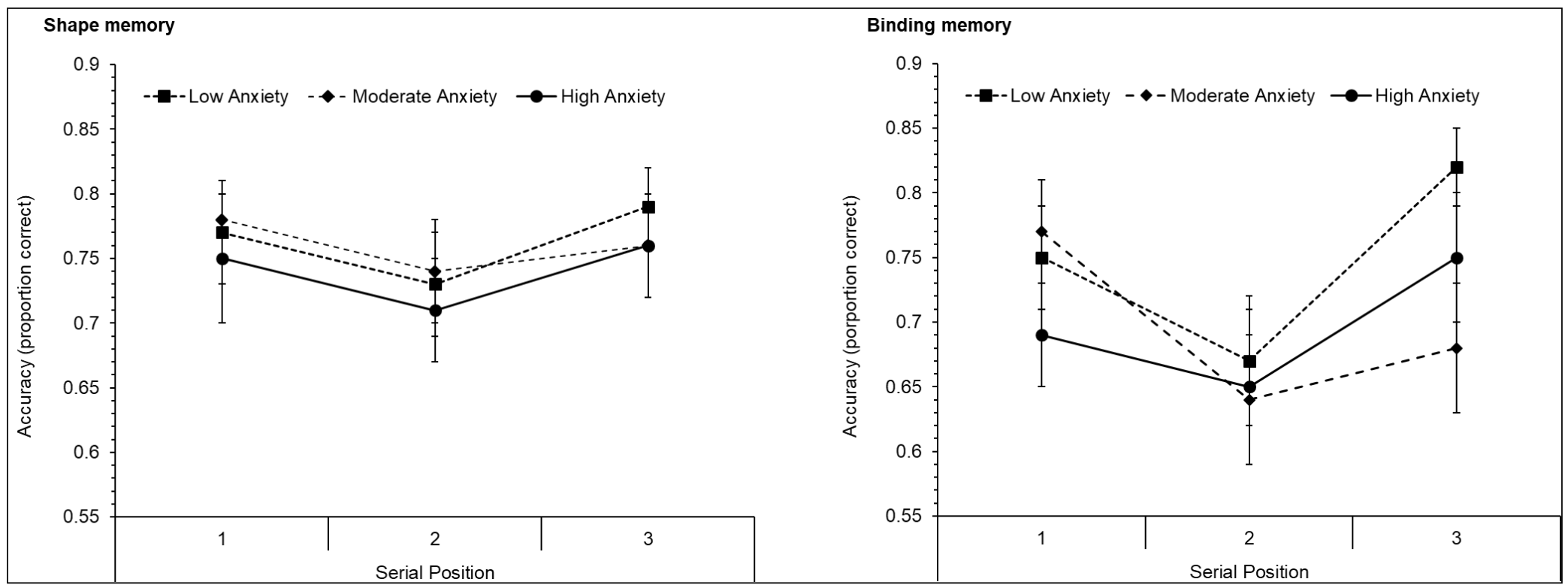




\section{Trait somatic anxiety}

Finally, to determine the specificity of the results to the cognitive dimension of trait anxiety, we used trait somatic anxiety scores to create the anxiety groups (see Table 1) and performed 3 (trait somatic anxiety group; low, moderate, high) x 2 (presentation format; simultaneous, sequential) x 2 (memory type; shape, binding) mixed factorial ANOVAs. Regarding accuracy, there were no significant effects involving anxiety (all $p$ involving anxiety $>.15$ ), including the two-way interaction between presentation type and trait somatic anxiety group, $F(2,88)=3.01, M S E=$ $.006, p=.054, \eta_{p}^{2}=.06$. Similarly, there were no significant effects involving trait somatic anxiety when analysing correct $\mathrm{RT}$ data (all $p>.53) .^{5}$

\section{Discussion}

As expected, a general binding deficit was associated with sequential presentation of visual stimuli, confirming the vulnerability of visual bindings in working memory, and their susceptibility to interference by further stimuli (Allen et al., 2006; Allen et al., 2014; Baddeley et al., 2011; Brown \& Brockmole, 2010). With the sequences containing three objects, it is notable that those presented at the middle position were relatively vulnerable in working memory. This pattern is consistent with earlier work using the same sequence length (Brown et al., 2017; Allen, Atkinson, \& Nicholls, 2020). Generally, sequential presentation is challenging because it requires working memory resources, including internal/goal-directed attention, to be

\footnotetext{
${ }^{5}$ Multilevel models indicated that there were no significant effects involving trait somatic anxiety either for accuracy (all $p$ involving anxiety $>.20$ ) or for correct RT (all $p>.32$ ).
} 
redistributed in order to encode newer stimuli (Gorgoraptis et al., 2011), while also retaining earlier items (Hitch, Allen, \& Baddeley, 2020).

Regarding a potential role of trait anxiety in visual working memory performance, an intriguing interaction was observed between presentation format and trait cognitive anxiety on efficiency (RT during correct responses). The moderate anxiety group performed more efficiently during sequential compared to simultaneous presentation, for both individual feature and binding memory. Based on ACT (Eysenck et al., 2007), higher trait anxiety may be associated with reduced performance efficiency. Interestingly, in the high trait cognitive anxiety group, RT did appear numerically higher in the sequential compared with the simultaneous condition, but this difference was not reliable, and so we cannot conclude that high anxiety reduces efficiency where there is increased demand (Berggren \& Derakshan, 2013). Our finding of increased efficiency in the moderate anxiety group is, however, apparently related to the enhanced attentional demand of sequential presentation conditions. The effect is unlikely to be an artefact of increased guessing, given that we assessed RT during correct trials only. Rather, it may reflect that these participants experienced an additional attentional demand involved in retaining the earlier items in each trial while also encoding the new ones, resulting in increased effort to meet that demand (Ansari \& Derakshan, 2011). Interestingly, visual detection (Berggren et al., 2015) and visual working memory capacity (Moriya, 2018; Moriya \& Sugiura, 2012; see also Moran, 2016) have occasionally been positively associated with anxiety in previous research, and the present findings may be a reflection of a potential enhancement for processing and retaining visual material, in some circumstances. The precise task demands as well as the specific levels of anxiety within participant samples therefore has potential to reveal benefits in 
visual working memory. Notably, this effect was only observed with trait cognitive anxiety, however, and not trait somatic anxiety.

Regarding performance effectiveness (accuracy), the predicted pattern of performance was observed in the form of progressively lower accuracy from low through moderate and high levels of trait cognitive anxiety. However, this pattern was not reliable and the results therefore did not support the hypothesis, that higher trait cognitive anxiety would be associated with poorer binding performance under sequential presentation conditions. This was the case also when considering trait somatic anxiety. These findings do however support previous research showing no behavioural effects of increased anxiety or worry on visual working memory accuracy (e.g. Moreno et al., 2015; Qi, Chen, et al., 2014). Based on the present findings, then, visual working memory may not be as directly vulnerable to the effects of trait anxiety on attention as other working memory functions such as inhibition, attentional shifting, or spatial working memory (Moran, 2016).

\section{Study 2}

According to ACT, trait anxiety interacts with situation-specific stressors to predict state anxiety (Eysenck et al., 2007; Eysenck \& Derakshan, 2011). Recent research has therefore investigated the impacts of trait anxiety on working memory by looking specifically at the effects of induced stressful states. Sari, Koster, and Derakshan (2017) investigated the impacts of worry on change detection performance, by asking participants to think of a personal concern or upcoming worrisome event prior to task completion. The worry manipulation did not directly affect working memory capacity, but the worry condition and not the control condition was associated 
with impaired working memory capacity indirectly, through state worry and state anxiety. Similarly, Edwards et al. $(2015,2017)$ investigated potential impacts of anxiety on executive functions. They manipulated stress by informing participants that the upcoming tasks were indicative of intelligence, and that they were performing worse than expected. Edwards et al. (2015) found that, at higher mental effort, higher cognitive trait anxiety was associated with poorer attentional shifting efficiency. At lower mental effort, though, the relationship was stronger and most pronounced when participants had experienced situational stress.

It is therefore also important to assess the potential interaction between trait anxiety and induced mood state on visual working memory, and previous authors have specifically noted the requirement for research using this approach (e.g. Berggren et al., 2017; Moriya \& Sugiura, 2012). In Study 2 we focused on assessing working memory for simultaneously presented shapes and feature bindings presented simultaneously, and investigated the potential impact of a stress manipulation procedure. The simultaneous presentation format was chosen rather than sequential, in order to assess effects independently of increased task demand (Eysenck et al., 2007; Eysenck \& Derakshan, 2011) and, given the potential effect of stress, to avoid floor effects in task performance.

Situational stress was expected to increase state anxiety, especially in those with higher trait anxiety, in turn reducing availability of executive attentional resources. We therefore predicted that visual working memory would be negatively impacted by higher trait anxiety, specifically in the situational stress condition, and for binding more than shape memory. Again, to test our prediction that trait cognitive anxiety is the specific component that will impact task 
performance, it was necessary also to test the effects of the somatic component (e.g. Edwards et al., 2017).

\section{Method}

\section{Participants}

After excluding one participant due to missing data, there were 111 young adult participants (63 females), aged 18-35 years (see Table 3). Participants were recruited from the Psychology undergraduate participant pool at the University of Strathclyde, and via opportunity sampling. This sample size surpassed that of similar previous studies that included more predictor variables (e.g., Edwards et al., 2015, 2017). Participants from the pool were awarded course credit for participation. All participants reported normal or corrected vision, and no memory impairments.

Table 3. Baseline trait anxiety measures, age, years of education (all with $S D$ s), and sex distribution for Study 2 participants, overall and by situational stress condition.

\begin{tabular}{|c|c|c|c|c|c|c|c|}
\hline & $\mathrm{N}$ & $\begin{array}{c}\text { Age } \\
M( \pm S D)\end{array}$ & $\begin{array}{c}\text { Sex } \\
(M: F)\end{array}$ & $\begin{array}{c}\text { Yrs } \\
\text { Education } \\
M( \pm S D)\end{array}$ & $\begin{array}{l}\text { Overall Trait } \\
\text { Anxiety } \\
\text { Min-Max, M } \\
( \pm S D)\end{array}$ & $\begin{array}{c}\text { Trait Cognitive } \\
\text { Anxiety } \\
\text { Min-Max, M } \\
( \pm S D)\end{array}$ & $\begin{array}{l}\text { Trait Somatic } \\
\text { Anxiety } \\
\text { Min-Max, M } \\
( \pm S D)\end{array}$ \\
\hline Overall & 111 & $\begin{array}{l}22.09 \\
(2.46)\end{array}$ & $48: 63$ & $\begin{array}{l}15.68 \\
(1.66)\end{array}$ & $\begin{array}{c}25-70,39.41 \\
(10.01)^{\star}\end{array}$ & $\begin{array}{c}11-38,21.28 \\
(5.85)\end{array}$ & $\begin{array}{c}11-36,18.12 \\
(5.25)^{*}\end{array}$ \\
\hline Control & 56 & $\begin{array}{l}22.14 \\
(2.58)\end{array}$ & $27: 29$ & $\begin{array}{l}15.80 \\
(1.66)\end{array}$ & $\begin{array}{c}26-64,40.18 \\
(9.82)\end{array}$ & $\begin{array}{c}13-38,21.71 \\
(5.54)\end{array}$ & $\begin{array}{c}11-31,18.46 \\
(5.18)\end{array}$ \\
\hline Stress & 55 & $\begin{array}{l}22.04 \\
(2.34)\end{array}$ & $21: 34$ & $\begin{array}{l}15.55 \\
(1.66)\end{array}$ & $\begin{array}{c}25-70,38.61 \\
(10.24)^{\star}\end{array}$ & $\begin{array}{c}11-34,20.84 \\
(6.18)\end{array}$ & $\begin{array}{c}11-36,17.76 \\
(5.36)^{*}\end{array}$ \\
\hline
\end{tabular}

${ }^{*}$ Based on N-1, due to one missing trait somatic anxiety score. 


\section{Design}

We assessed the extent to which trait anxiety, situational stress (control/stress), memory type (shape/binding), and the interactions, could predict memory performance, in terms of both accuracy (effectiveness) and RT in correct trials (efficiency) outcomes. Trait cognitive and somatic anxiety were assessed as predictors in separate analyses.

\section{Materials}

Anxiety measure: The STICSA (Ree et al., 2008) was the measure of trait cognitive and somatic anxiety and was used to evaluate the efficacy and impacts of the stress induction procedure over time, regarding state anxiety scores.

Visual working memory task: The task and stimuli were the same as for the simultaneous presentation condition of Study 1 (see top of Figure 1), except that the memory array presentation time was $500 \mathrm{~ms}$ in total, similar to previous research using simultaneous presentation only (e.g. Allen et al., 2006; Brown \& Brockmole, 2010). Also, there was a separate, additional 'practice' block of eight trials. Half of these tested shape and binding memory each, and half featured probes that had been presented in the memory array. Participants were advised that this was a short practice, but it was in fact to facilitate the situational stress induction.

Situational stress induction: The situational stress induction procedure followed an ego threat method (e.g. Edwards et al., 2015, 2017). In the stress group, at the start of the 'practice' trials, participants were informed that: 1) performance in the task was related to intelligence; 2) 
at the end of the whole session, they would be advised of their estimated intelligence score, calculated on the basis of their performance; and 3) they would receive an update on their performance after the practice trials were completed. Following the practice trials, and regardless of performance level, all participants in the stress condition were advised that, so far, their performance level was 'somewhat slower and less accurate than others in the study'. Control participants also performed the practice trials, but there was no reference to intelligence at any point.

\section{Procedure}

Prior to data collection, ethical approval was obtained from the School of Psychological Sciences \& Health Ethics Committee at the University of Strathclyde. Given the situational stress manipulation, it is important to specify that, as would be expected, all participants were fully debriefed immediately after data collection and reminded of the possibility of withdrawing their data. No participants did so.

All participants completed the STICSA-Trait then STICSA-State questionnaires, prior to the cognitive task. Following the 'practice' trials (and the situational stress induction, for those assigned to that condition), all participants completed the STICSA-State again. There were then two counterbalanced blocks of trials, testing shape and binding memory. As in Study 1, 
participants performed articulatory suppression during performance of each trial. ${ }^{6}$ The STICSAState questionnaire was administered for a third time upon completion of all trials, then participants were immediately debriefed.

\section{Results}

To assess the impact of the stress induction, overall state anxiety scores were entered into a 2 (control, stress) x 3 (time; session start, post-practice, session end) mixed ANOVA (see Figure 5). ${ }^{7}$ There was no main effect of stress $(p=.87)$, but the effect of time, $F(2,200)=35.11, M S E=$ $15.96, p<.001, \eta_{p}^{2}=.26$, and the interaction, $F(2,200)=11.72, p<.001, \eta_{p}^{2}=.11$, were significant. The effect of time was stronger in the stress group, $F(2,110)=47.81, M S E=14.73$, $p<.001, \eta_{p}^{2}=.48$, than the control group, $F(2,98)=3.23, M S E=17.24, p=.044, \eta_{p}^{2}=.06$. Planned comparisons showed that, in the control group, state anxiety was not significantly different between times $1(M=34.22, S D=8.50)$ and $2(M=35.08, S D=8.62 ; t(49)=.97, p=$ $.34)$ or between times 2 and $3(M=36.51, S D=8.35 ; t(50)=1.54, p=.13)$. However, in the stress group, state anxiety was lower at time $1(M=31.38, S D=7.65)$ than time $2(M=36.46$, $S D=8.95 ; t(51)=6.98, p<.001)$, and at time 2 compared with $3(M=38.54, S D=10.15 ; t(51)$ $=2.76, p=.008)$.

\footnotetext{
${ }^{6}$ Articulation rate was again very consistent across all conditions, with a grand mean of $5.42(S D=.87)$. A 2 (situational stress condition; control/stress) x 2 (memory type; shape/binding) mixed ANOVA showed no significant effects (all $p>.06$ ).

${ }^{7}$ Due to missing state anxiety data, $n=50$ for the control group, and $n=52$ for the stress group.
} 
Figure 5. Overall state anxiety scores across the testing session in Study 2. Scores were taken at session start (following the trait anxiety questionnaire), then after the memory task practice block (including the situational stress manipulation for those in the experimental condition), and finally after completing the memory task.

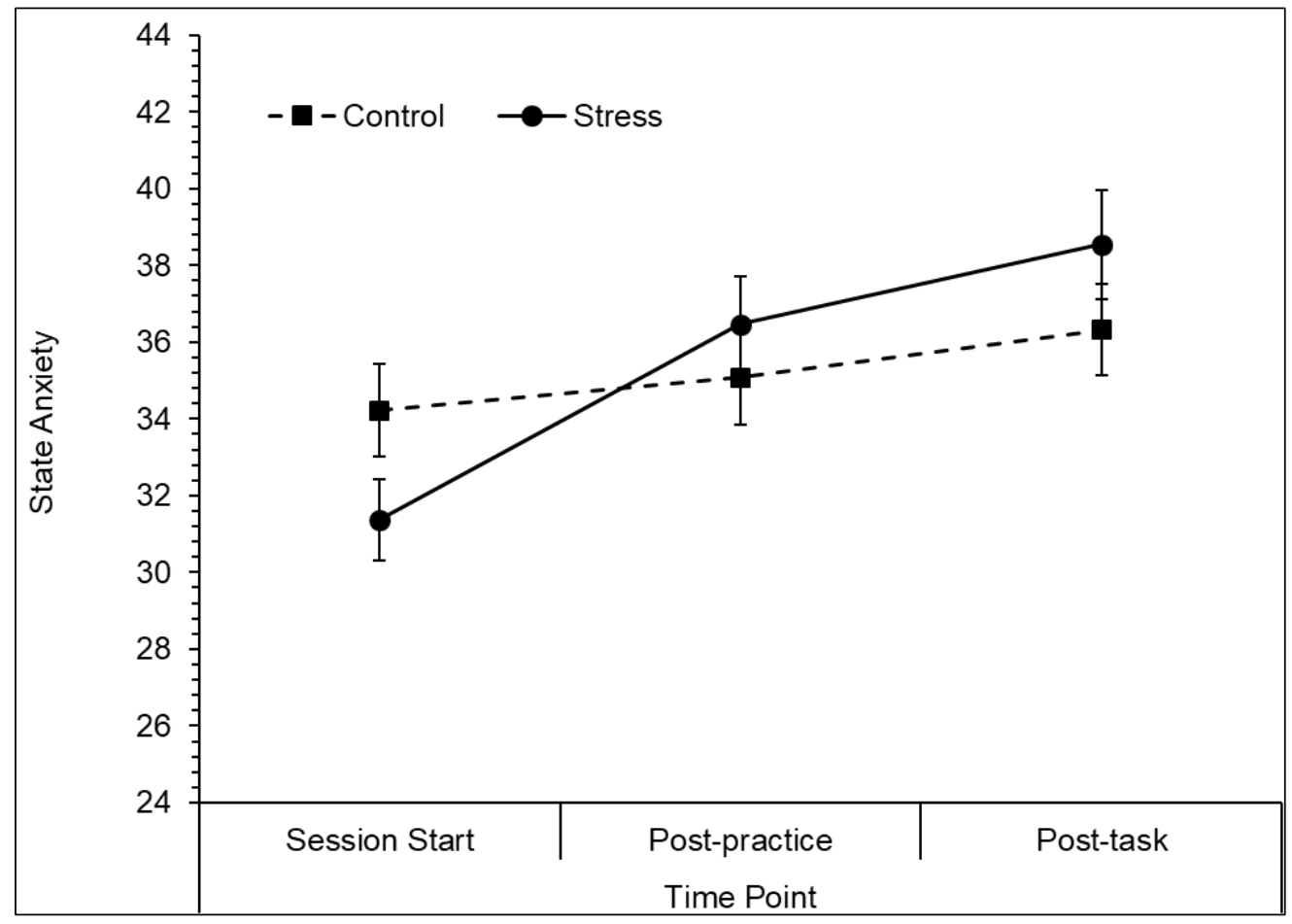

As participants were initially randomly assigned to different stress conditions, it was not possible to group the sample evenly according to anxiety scores as in Study 1, while also taking situational stress into account. Thus, multilevel mixed modelling was more appropriate to assess whether response accuracy (effectiveness) and correct RT (efficiency) were predicted by situational stress condition, memory type, mean-centred trait anxiety scores, and their interactions. Participants' anxiety scores, stress condition (control, stress), and memory type (shape, binding) were each considered fixed-effect predictors. Terms were entered factorially, at a single level, including each main effect, the two-way interactions, and the three-way 
interaction. An unstructured repeated covariance structure was selected, in line with multivariate repeated-measures ANOVA (Hoffman \& Rovine, 2007). Participant identifier served as a random factor. Separate analyses included trait cognitive and trait somatic anxiety (Edwards et al., 2017).

Where multilevel modelling demonstrated significant effects, follow-up analyses were then considered and conducted where appropriate, to assess the extent to which state anxiety may have mediated the relationships observed. Mediation analyses were conducted using the PROCESS path analysis macro for SPSS (Hayes, 2017). Based on the pattern of state anxiety scores across testing sessions (Figure 5), and the Pearson correlations reported in Table 4, state anxiety levels at the post-task phase (time 3) were selected as potential mediators. Analyses were based on 5000 bootstrapped samples. ${ }^{8}$

\footnotetext{
${ }^{8}$ Due to missing state anxiety data, for the mediation analyses $n=104$ or 105 , depending on the analysis.
} 
Table 4. Correlations amongst state anxiety measures at time points 2 (post-practice/stress manipulation) and 3 (post-working memory task), and the outcomes significantly predicted by IVs in the multilevel models.

\begin{tabular}{|c|c|c|c|c|c|c|c|c|c|c|c|}
\hline & 1 & 2 & 3 & 4 & 5 & 6 & 7 & 8 & 9 & 10 & 11 \\
\hline 1. Trait anxiety & - & & & & & & & & & & \\
\hline 2. Trait cog. anxiety & $.91^{* *}$ & - & & & & & & & & & \\
\hline 3. Trait som. anxiety & $.89^{* *}$ & $.62^{* *}$ & - & & & & & & & & \\
\hline 4. State anxiety (post-practice) & $.55^{\star *}$ & $.53^{* *}$ & $.44^{* *}$ & - & & & & & & & \\
\hline 5. State cog. anxiety (post-practice) & $.60^{* *}$ & $.66^{* *}$ & $.38^{* *}$ & $.88^{* *}$ & - & & & & & & \\
\hline 6. State som. anxiety (post-practice) & $.30^{*}$ & $.20^{*}$ & $.33^{*}$ & $.81^{* *}$ & $.43^{* *}$ & - & & & & & \\
\hline 7. State anxiety (post-task) & $.64^{* *}$ & $.65^{\star \star}$ & $.46^{* *}$ & $.82^{* *}$ & $.80^{\star *}$ & $.56^{* *}$ & - & & & & \\
\hline 8. State cog. anxiety (post-task) & $.62^{* *}$ & $.71^{* *}$ & $.37^{* *}$ & $-.72^{* *}$ & $.86^{* *}$ & $.32^{*}$ & $.90^{* *}$ & - & & & \\
\hline 9. State som. anxiety (post-task) & $.46^{\star *}$ & $.38^{* *}$ & $.44^{* *}$ & $.68^{* *}$ & $.48^{\star *}$ & $.68^{* *}$ & $.83^{\star \star}$ & $.49^{\star \star}$ & - & & \\
\hline 10. Stress condition & -.08 & -.08 & -.07 & .07 & .03 & .06 & .11 & .04 & .17 & - & \\
\hline 11. Binding accuracy & -.17 & -.11 & $-.19^{*}$ & -.08 & -.16 & .08 & $-.24^{*}$ & $-.27^{\star}$ & -.13 & $-.22^{*}$ & - \\
\hline 12. Correct RT & $.25^{*}$ & $.22^{*}$ & $.23^{*}$ & .16 & .09 & .18 & .19 & .11 & $.23^{*}$ & -.10 & .07 \\
\hline
\end{tabular}




\section{Trait cognitive anxiety}

Parameter estimates for models based on trait cognitive anxiety can be viewed in Table 5. For the multilevel mixed model predicting performance effectiveness (i.e. accuracy), memory type significantly predicted accuracy, $F(1,107)=12.78, p=.001$, with shape memory more accurate than binding memory $b=-0.035$ CI $[-.055,-.016], \mathrm{t}(107)=-3.57, p=.001$. The interaction between stress condition and memory type was also a significant predictor, $F(1,107)=6.74, p=$ .011. The interaction was followed-up by conducting multilevel modelling of the effect of stress condition within each memory type condition, excluding the anxiety variable. There was no significant effect of stress on shape memory, $F(1,110)=0.05, p=.82$, but a significant effect on binding memory, $F(1,110)=5.49, p=.021$, in which performance was poorer in the stress than the control condition, $b=-0.043,95 \%$ CI [-.079, -.007], $t(110)=-2.34, p=.021$. This effect was not followed-up via mediation analysis, as there were no significant correlations between stress condition and state anxiety measures (see Table 4). Finally, in the multilevel model, trait cognitive anxiety did not predict performance accuracy, or significantly interact with any other variables, including the interaction between trait cognitive anxiety and memory type, $F(1,107)=$ $3.67, p=.058($ all other $p>.19)$. 
Table 5. Unstandardised coefficients, and 95\% confidence intervals for each multilevel model involving trait cognitive anxiety in Study 2.

\begin{tabular}{lcccc}
\hline & Unstandardised Coefficients & \multicolumn{2}{c}{$\begin{array}{c}95 \% \text { Confidence Interval } \\
\text { for B }\end{array}$} \\
\cline { 2 - 5 } & & $S E$ & Lower & Upper \\
& & & Bound & Bound \\
\hline Effectiveness (accuracy) & & & \\
Trait cognitive anxiety & & & & .002 \\
Stress condition & -.001 & .001 & -.003 & .011 \\
Memory type & -.021 & .016 & -.052 & -.016 \\
Trait cognitive anxiety x stress & -.035 & .010 & -.055 & .006 \\
Trait cognitive anxiety x memory type & .001 & .003 & -.005 & .000 \\
Stress x memory type & -.003 & .002 & -.007 & -.012 \\
Trait cognitive anxiety x stress x memory type & -.051 & .020 & -.090 & .011 \\
\hline Efficiency (correct RT) & .004 & .003 & -.002 & 13.48 \\
Trait cognitive anxiety & & & & 38.92 \\
Stress condition & 7.00 & 3.27 & 0.52 & 47.31 \\
Memory type & -36.21 & 37.90 & -111.34 & 18.63 \\
Trait cognitive anxiety x stress & 17.24 & 15.17 & -12.83 & -7.29 \\
Trait cognitive anxiety x memory type & 5.67 & 6.54 & -3.94 & 6.43 \\
Stress x memory type & 1.25 & 2.62 & -86.98 & 33.28 \\
Trait cognitive anxiety x stress x memory type & -26.85 & 30.33 & -8.54 & 12.20 \\
\hline
\end{tabular}


Table 6. Unstandardised coefficients and $95 \%$ confidence intervals for each multilevel model involving trait somatic anxiety in Study 2.

\begin{tabular}{|c|c|c|c|c|}
\hline & \multicolumn{2}{|c|}{ Unstandardised Coefficients } & \multicolumn{2}{|c|}{$\begin{array}{c}\text { 95\% Confidence Interval } \\
\text { for B }\end{array}$} \\
\hline & $\mathrm{B}$ & SE & Lower & Upper \\
\hline & & & Bound & Bound \\
\hline \multicolumn{5}{|l|}{ Effectiveness (accuracy) } \\
\hline Trait somatic anxiety & -.002 & .002 & -.005 & .001 \\
\hline Stress condition & -.020 & .016 & -.051 & .011 \\
\hline Memory type & -.035 & .010 & -.054 & -.016 \\
\hline Trait somatic anxiety $\mathrm{x}$ stress & .001 & .003 & -.005 & .007 \\
\hline Trait somatic anxiety $x$ memory type & -.004 & .002 & -.008 & -.001 \\
\hline Stress $\mathrm{x}$ memory type & -.048 & .020 & -.087 & -.010 \\
\hline Trait somatic anxiety $x$ stress $x$ memory type & -.002 & .004 & -.009 & .005 \\
\hline \multicolumn{5}{|l|}{ Efficiency (correct RT) } \\
\hline Trait somatic anxiety & 8.74 & 3.64 & 1.53 & 15.95 \\
\hline Stress condition & -32.43 & 38.03 & -107.83 & 42.97 \\
\hline Memory type & 13.67 & 14.86 & -15.79 & 43.14 \\
\hline Trait somatic anxiety $x$ stress & 1.54 & 7.27 & -12.88 & 15.96 \\
\hline Trait somatic anxiety $x$ memory type & 5.10 & 2.84 & -0.54 & 10.73 \\
\hline Stress $\mathrm{x}$ memory type & -27.91 & 29.73 & -86.85 & 31.02 \\
\hline Trait somatic anxiety $x$ stress $x$ memory type & -8.18 & 5.68 & -19.45 & 3.10 \\
\hline
\end{tabular}

Regarding the model using efficiency (i.e. correct RT) as the outcome variable, trait cognitive anxiety significantly predicted correct RT, $F(1,107)=4.59, p=.035$, with RTs increasing as anxiety increased, $\mathrm{b}=6.99 \mathrm{CI}[.52,13.48], t(107),=2.14, p=.035$. There were no other significant effects (all $p>.25$ ). Mediation analysis including state somatic anxiety as the mediator (as this was significantly correlated with overall correct RT; see Table 4), indicated that there was a total effect of trait cognitive anxiety, $b=8.51,95 \%$ CI $[1.87,15.15], t=2.54, p=$ 
.013 , confirming that this was a significant predictor. Trait cognitive anxiety predicted state somatic anxiety, $b=.31,95 \% \mathrm{CI}[.16, .46], t=4.11, p<.001$, but the direct, $b=6.45,95 \%$ CI [$.67,13.57], t=1.80, p=.075$, and indirect, $b=2.06,95 \% \mathrm{CI}[-.82,5.38]$ effects were not significant. Therefore, while trait cognitive anxiety was associated with both state somatic anxiety and correct RT, the relationship between trait cognitive anxiety and correct RT was not mediated by state somatic anxiety.

\section{Trait somatic anxiety}

For models based on trait somatic anxiety, parameter estimates can be viewed in Table 6 . In addition to the above noted significant effect of memory type $(p<.001)$ and interaction between stress condition and memory type $(p=.015)$, the interaction between trait somatic anxiety and memory type also predicted effectiveness, $F(1,106)=5.80, p=.018$ (all other $p>.21$ ). This was followed up with multilevel modelling of the effect of trait somatic anxiety within each memory task type, excluding stress condition. Trait somatic anxiety did not predict shape memory accuracy $(p=.728)$, but did significantly predict binding accuracy, $F(1,108)=4.08, p=.046$, which decreased with increasing anxiety, $b=-.004,95 \%$ CI $[-.007,-.000], t(108)=-2.02, p=$ .046. Mediation analysis, including state cognitive anxiety as the mediator (see Table 4), showed that there was no significant total effect of trait somatic anxiety, $b=-.003,95 \%$ CI [-.007, -.001], $t=-1.43, p=.155$, while both the direct, $b=-.001,95 \%$ CI $[-.005, .003], t=-0.53, p=.60$, and indirect effects, $b=-.002,95 \%$ CI [-.004, .000], were not significant. However, trait somatic anxiety predicted state cognitive anxiety, $b=.44,95 \%$ CI $[.22, .66], t=4.00, p<.001$, and both variables interacted to predict binding accuracy, $F(1,100)=4.41, p=.038$. Thus, while trait 
somatic anxiety was associated with state anxiety, it did not predict binding accuracy directly or indirectly, with its predictive effect stemming from an interaction with state anxiety (i.e. a moderating effect). Moderated regression including each of these variables (mean-centred) and their interaction term confirmed that this interaction was significant, $b=-.00195 \%$ CI [-.001, $.000], t=-2.10, p=.038$. In Figure 6, binding accuracy can be viewed as a function of trait somatic anxiety and state cognitive anxiety (with low and high levels defined as $1 S D$ below and above the mean, respectively; Dawson, 2014). This demonstrates that, specifically in those with higher trait somatic anxiety, binding accuracy decreased with higher state cognitive anxiety.

Figure 6. The interaction between trait somatic anxiety and state cognitive anxiety which predicts binding accuracy in Study 2.

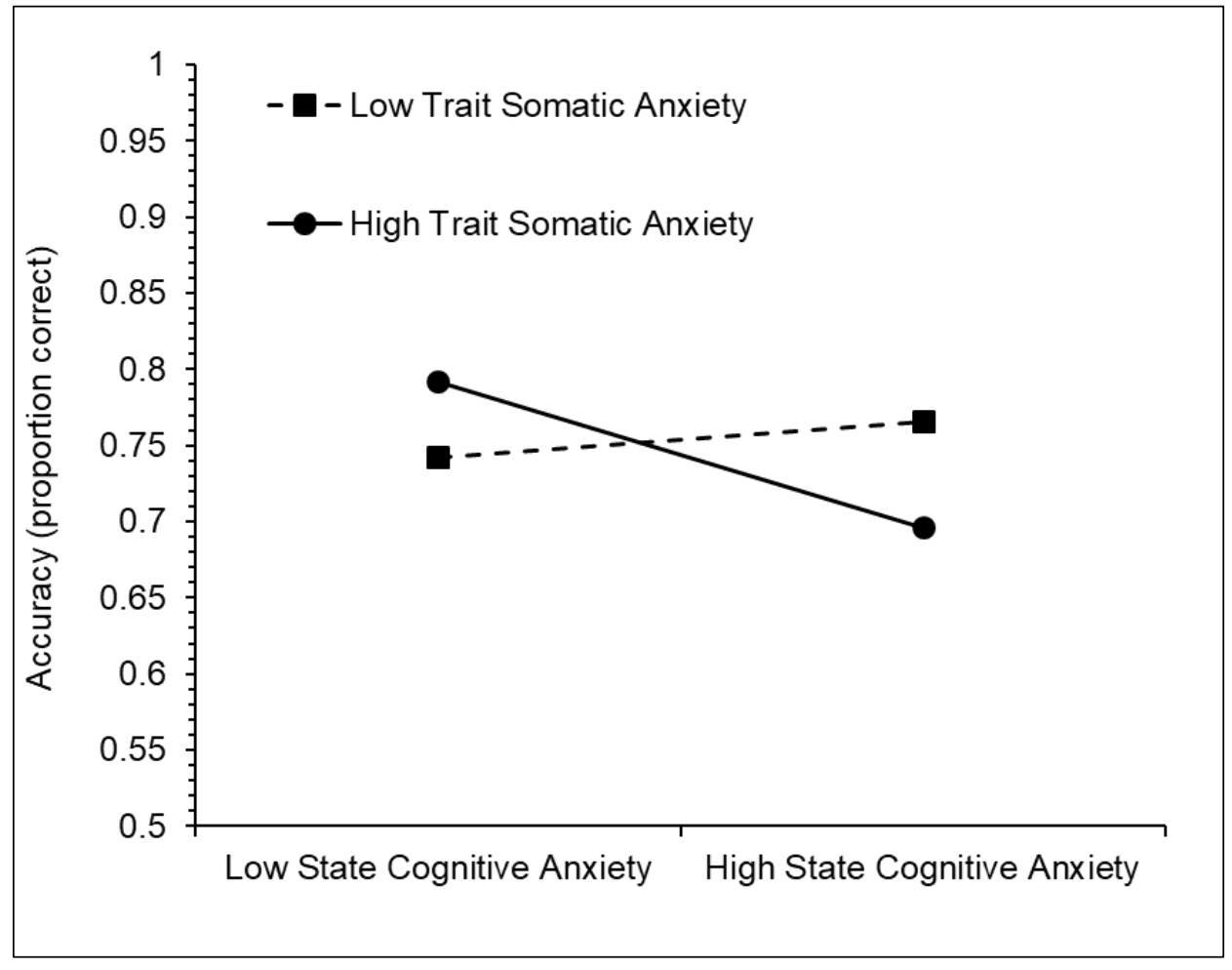


Trait somatic anxiety also significantly predicted efficiency, $F(1,106)=5.78, p=.018$, with overall RT increasing with anxiety score, $b=8.74,95 \%$ CI $[1.53,15.95], t(106)=2.40, p=$ .018. There were no other significant main effects or interactions, including the interaction between trait somatic anxiety and memory type, $F(1,106)=3.22, p=.076($ all other $p>.15)$. Mediation analysis indicated that there was a total effect of trait somatic anxiety, $b=10.88,95 \%$ CI $[3.17,18.58], t=2.80, p=.006$. Trait somatic anxiety predicted state somatic anxiety, $b=.42$, 95\% CI $[.25, .59], t=4.91, p<.001$, but there was no significant direct effect, $b=8.18,95 \% \mathrm{CI}$ $[-0.34,16.71], t=1.90, p=.060$ or indirect effect, $b=2.69,95 \%$ CI $[-1.86,6.90]$ on correct RT. Therefore, while trait somatic anxiety was associated with both state somatic anxiety and correct RT, the relationship between trait somatic anxiety and correct RT was not mediated by state somatic anxiety.

\section{Discussion}

Significant task manipulation effects were observed in Study 2, as expected. As in Study 1, visual feature binding was shown to be more challenging than individual feature (shape) memory when considering accuracy, again demonstrating that binding is not cost-free (e.g. Brown et al., 2017). In terms of situational stress, not only did this increase state anxiety levels over the course of the testing session, but the stress condition was also associated with reduced accuracy specifically in the more challenging binding memory condition.

Regarding anxiety, in line with the predictions and the results of Study 1, shape memory effectiveness (accuracy) was not predicted by trait cognitive anxiety, or indeed trait somatic anxiety or situational stress. This supports ACT inasmuch as shape memory is lower in cognitive 
demand, with higher demand tasks expected to be more sensitive to the effects of anxiety (Eysenck et al., 2007; Eysenck \& Derakshan, 2011). However, in contrast, binding effectiveness was predicted by trait somatic anxiety, and, additionally, both dimensions of trait anxiety predicted general task efficiency (correct RT). Study 2 therefore highlights relationships between trait anxiety and visual working memory performance efficiency and effectiveness, even with the neutral stimuli presently used. Furthermore, these relationships are not necessarily more strongly related to the cognitive dimension of trait anxiety. Moreno et al. (2015) specifically considered worry, a cognitive feature of anxiety, and found no greater binding deficit in high versus low worriers during a simultaneous binding task. However, they did observe a relationship between increased worry and response time in visuo-spatial working memory generally, consistent with the present effect on general task efficiency. Of particular interest here is that we observed this effect both with trait cognitive and somatic anxiety, meaning that the effect generalises beyond cognitive anxiety/worry.

Our results are therefore at odds with previous research examining both cognitive and somatic dimensions of anxiety, and which have not found significant associations between somatic anxiety and inhibitory control (Edwards et al. 2017), phonological processing efficiency (Edwards, Edwards \& Lyvers, 2016), or measures of processing speed, cognitive flexibility and verbal working memory in older adults (Mella et al., 2018). Observed associations between working memory/executive function deficits and physiological factors such as chronic pain (Berryman et al., 2013; 2014) and chronic fatigue syndrome (e.g. Joyce, Blumenthal, \& Wessely, 1996; Marshall, Forstot, Callies, Peterson, \& Schenck, 1997) suggest that reduced cognitive functioning may be related to somatic symptomology. However, thus far, specifically somatic 
anxiety has not been tested extensively. More research is therefore required to investigate further the relationship between somatic anxiety and working memory performance, which was clearly observed presently both in terms of binding effectiveness, and general visual working memory efficiency. Importantly, we observed that trait somatic anxiety interacted with state cognitive anxiety to predict binding effectiveness. That is, there was an effect of state cognitive anxiety on binding accuracy specifically in those with higher trait somatic anxiety. This shows that, although the somatic dimension was the significant predictor at the trait level, the relationship with binding accuracy was moderated by the cognitive dimension of state anxiety. This highlights both the interactive relationship between trait and state anxiety (Eysenck et al., 2007) for influencing task effectiveness in the current study, and the importance of specifically state cognitive anxiety regarding capacity limits on working memory resources. There is some previous evidence to suggest that trait anxiety may be more likely to influence cognitive performance when interacting with state anxiety. Egloff and Hock (2001), for example, found a positive relationship between emotional Stroop interference and state anxiety in a high trait anxious group, but a negative relationship in a low trait anxious group. This effect has also been observed when state anxiety was experimentally induced, with high trait anxious individuals showing greater interference from negative words under negative mood induction, and greater interference from positive words under positive mood induction, with no such effect in a low anxious group (Richards, French, Johnson, Naparstek, \& Williams, 1992; although see Mogg, Mathews, Bird, \& MacGregor-Morris, 1990). Our results therefore present an unexpected but potentially valuable explanation of the specific anxiety dimensions underlying the interaction of trait and state anxiety and the subsequent impact of this on cognitive performance. Somatic symptoms are perhaps best reflective of trait susceptibility to anxious states, with situational 
cognitive anxiety then exacerbating effects of trait anxiety. This interactive relationship should be explored in future research.

In terms of situational stress, although this separately predicted binding effectiveness, this never interacted with trait anxiety to predict task performance. Thus, stress did not bring about or exacerbate the impact of trait anxiety, even in the most demanding binding memory task. Furthermore, as confirmed via mediation analyses, stress did not appear to impact performance via state anxiety, at least as measured by the STICSA. It is possible that the cognitive task may need to make more specific demands on executive attention to observe an interaction with situational stress, and/or mediation by state anxiety, such as by involving inhibition or shifting (Edwards et al., 2015, 2017), or attentional control in response to cues (Sari et al., 2017; see also Eysenck et al., 2007). Another possibility is that, because state anxiety, by definition, varies moment-to-moment, the state anxiety measure would have been most sensitive to the manipulation if taken at another time point, such as mid-way during task performance. However, more questionnaire administrations would have been undesirable, as administration during or between performance of trial blocks could have been disruptive. As such, time-point 3 certainly appeared to be the peak, in terms of the effect of the stress manipulation on the state anxiety scores across groups, and was chosen on this basis. We speculate that, in our study, situational stress may have directly caused an executive demand that is not specifically due to increased state anxiety. Rather, the manipulation may have taken up executive resources due to more task monitoring/consideration of the information provided (i.e. intelligence level and the relationship with the task) across all participants. In short, the effect of situational stress may have brought about a dual-task demand. Future research could therefore usefully assess the range of potential 
impacts of induced situational stress, including state anxiety as well as more generalised executive demand, in order to determine the underlying mechanism/s of any effect. Notably, participants' mental effort, which was not measured in the current study, may also have played a role (Edwards et al., 2017), and this could also be taken into account in future research alongside attentional demand and situational stress.

\section{General Discussion}

We investigated the extent to which trait anxiety affects visual working memory, a potential relationship which has received relatively little attention (Baddeley et al., 2012; Moran, 2016). In the context of Attentional Control Theory (ACT; Eysenck et al., 2007) and recent working memory models (Allen et al., 2017; Baddeley, 2012; Logie, 2011), we varied executive attentional demand (Study 1) and situational stress (Study 2). In Study 1 it was found that a moderate level of anxiety was associated with faster correct responses in the sequential (more demanding) relative to simultaneous binding condition, but there were no other significant effects observed relating to anxiety. In Study 2, involving simultaneous shape and binding memory only, under either control or situational stress conditions, higher trait cognitive and somatic anxiety predicted worse performance efficiency (correct RT). Situational stress and trait somatic anxiety also both predicted binding effectiveness (accuracy). However, no interactions were observed with situational stress, showing that this did not bring about or exacerbate the effect of anxiety (and vice versa). These findings demonstrate clear relationships between trait anxiety and visual working memory performance, and therefore some support for ACT (Eysenck et al., 2007). However, notably, it was in the relatively low demand scenario (i.e. Study 2, in 
which there was no specific additional attentional task demand), that both trait somatic anxiety and trait cognitive anxiety predicted performance, and not specifically the cognitive dimension).

Previous research addressing cognitive impacts of anxiety, in the context of multicomponent working memory models, has suggested that anxiety impacts memory via the central executive (Christopher \& MacDonald, 2005; Eysenck et al., 2005; Eysenck \& Derakshan, 2011), but not the phonological or visuospatial subsystems. The present studies drew upon recent research implicating central executive and perceptual factors in the binding of information in visual working memory. Study 1 reflected the expected pattern, based on the binding literature, whereby binding memory is significantly reduced compared to shape memory, and furthermore when stimuli are presented sequentially (Allen et al., 2006; Baddeley et al., 2011; Brown \& Brockmole, 2010; Brown et al., 2017; Gorgoraptis et al., 2011; Hu et al., 2014; Hu et al., 2016). The executive demand of the sequential binding task lies in the fact that participants must encode new objects into working memory while actively maintaining previous objects from the array (Allen et al., 2014, 2017). In Study 1, we therefore expected deficits to be greatest for participants in the high anxiety group, and potentially more generally at earlier serial positions, but these outcomes were not observed. While Figure 2 shows the predicted pattern of progressively reduced performance in the sequential binding task from low through to high trait cognitive anxiety, this was not significant. Therefore, on the basis of Study 1, we could not conclude that trait anxiety is related to visual working memory effectiveness. This was the case for both trait cognitive and somatic anxiety.

Regarding performance efficiency, however, the findings of Study 1 suggest that moderate levels of trait cognitive anxiety may be associated with improved performance efficiency at 
greater executive demand (i.e. sequential presentation). This finding is not in isolation in the literature, as there is evidence that high trait anxiety may facilitate performance in cognitive tasks (Berggren et al., 2015; Moriya, 2018; Moriya \& Sugiura, 2012; see also Moran, 2016) and may be related to enhanced, low-level visual processing under certain circumstances (Berggren et al., 2015). Yet, in Study 1, although not a reliable effect, efficiency was numerically poorer in the high trait cognitive anxiety group, and this group certainly did not show improved efficiency. Furthermore, in Study 2, higher trait anxiety was related to poorer visual working memory task efficiency (both for shape and binding memory), demonstrating the core prediction of limited cognitive efficiency with higher trait anxiety (Eysenck et al., 2007). This finding also supports those of Moreno et al. (2015), who found that response times were slower in a high worry group compared to low worry group for both shape and binding memory. Nonetheless, while both studies have in common the finding that performance efficiency in visual working memory is sensitive to trait anxiety, the observation in Study 1 merits more focused research in future. The boundary task conditions and stimuli, and specific forms and levels of anxiety that can potentially enhance visual perception and/or working memory performance remain to be determined.

\section{Dimensions of trait anxiety}

Studies of anxiety and visual working memory have thus far, to our knowledge, not analysed cognitive and somatic anxiety dimensions discretely. A key strength of the present research was therefore to consider the potential role of trait anxiety dimension. In both studies, cognitive trait anxiety did not significantly predict visual working memory effectiveness (see also Moreno et 
al., 2015). This is contrary to Edwards et al. (2017) who, in the context of executive functioning (inhibition), showed that trait cognitive anxiety, but not trait somatic anxiety, was predictive of efficiency and effectiveness. However, in the present Study 2, trait somatic anxiety predicted binding effectiveness (accuracy) and this was found to be moderated by state cognitive anxiety. Those with higher trait somatic anxiety exhibited an effect of state cognitive anxiety on binding accuracy. This suggests the interactive nature of both anxiety dimensions and that the cognitive impacts of anxiety are not exclusively related to a general propensity for cognitive indications of anxiety. Rather, in this case, the best predictor was somatic indications at trait level.

Further research is now required to investigate the potential effects of trait somatic anxiety on cognitive performance, as this outcome was inconsistent both with previous literature and, notably, with the current Study 1. First, it is important to highlight that reported anxiety levels were fairly consistent between the two studies and, in fact, Study 1 showed even higher maximum anxiety scores, so the observed anxiety levels cannot account for the disparity. It is possible that methodological differences may have impacted the results. For example, due to differences in the research designs, there was a reduced trial presentation time for the memory array in Study 2 (500ms) compared to Study 1 (750ms). This may also explain why trait cognitive anxiety and efficiency were linearly and negatively associated in Study 2, but not in Study 1, with less time available to encode stimuli potentially being an important factor in working memory deficits associated with anxiety. Also, the additional within-subjects factor in Study 1 meant that each participant carried out four full trial blocks in that study, but only two full blocks (albeit with an additional, shorter 'practice' trial block) in Study 2. With Study 2 demonstrating reliable, detrimental impacts of trait anxiety, there is potential, then, for task 
practice/session duration to interact with trait anxiety. Specifically, in shorter tasks, participants may not have sufficient time to adjust to the testing scenario both in terms of the task itself and the environment, amplifying trait anxiety effects. Future work should investigate this possibility, both within visual working memory but also in wider cognitive contexts.

Another possibility regarding a lack of effect of trait cognitive anxiety on performance effectiveness across both of our studies is that the vocal articulation required in repeating 2-digit numbers concurrently with the memory task may have limited any potential effects. It has previously been suggested that active worry in high anxious participants may serve as a secondary task in itself as it involves negative internal self-statements (e.g. Markham \& Darke, 1991). Thus, when introducing even a concurrent task that is not load-intensive (as with the articulatory suppression task used here), effects of worry may be masked as internal verbalisation of worries is not possible. That is, the load across anxiety levels becomes equivalent as it comes from the concurrent task, rather than any potential anxious cognitions. While articulatory suppression has been shown to be detrimental to performance in the verbal domain for highanxious participants, reducing comprehension in reading tasks (Calvo, 1996), it would be useful to extend comparisons between restricted and unrestricted articulation conditions to visual working memory tasks. Indeed, it appears that recent studies that have found effects of anxiety on visual working memory did not employ articulatory suppression (Berggren et al., 2017; Jaiswal et al., 2018). Our use of articulatory suppression was in keeping with most previous visual feature binding studies. Yet, comparing performance with and without articulatory suppression could help to indicate whether it is specifically verbal thoughts relating to worry or 
apprehension that may impact on working memory accuracy in the visual domain, and indeed whether task features could potentially distract from this.

Edwards et al. (2017) observed that situational stress interacted with trait cognitive anxiety, highlighting that a shared cognitive mechanism likely underlies this relationship. The present Study 2 showed an effect of situational stress that was not mediated by state anxiety, but which interacted with memory task type. This may be underpinned by taxing executive resources in a more dual-task fashion rather than via increased anxiety (e.g. increased task monitoring). Future work should investigate further the mechanism underlying the trait somatic anxiety-visual working memory association, as well as the cognitive impacts of situational stress, as this will have implications for developing ACT (Eysenck et al., 2007), in terms of the wider impacts of trait anxiety on working memory.

To further investigate and clarify the effects of trait anxiety on visual working memory performance, we believe future work should take the two anxiety dimensions into account, while also establishing the effects of different forms of attentional demand (e.g. dual-tasking, distractor inhibition; Brown \& Brockmole, 2010; Brown et al., 2017). Likewise, as discussed earlier, the potentially broader attentional impacts of situational stress, mood induction techniques, or even the working memory task itself should also be considered. The impact of irrelevant stimuli may be another useful avenue for exploration, as initial evidence has demonstrated the disruptive effect of irrelevant information on the visual working memory of high-anxious individuals (Berggren et al., 2015; Moser et al., 2012; Sadeh \& Bredemeier, 2011). This would be particularly interesting, given that to-be-ignored objects have been shown specifically to disrupt bindings (Hu et al., 2014; Hu et al., 2016). Studies using similar variables to predict executive 
functioning (Edwards et al., 2015, 2017) have also shown that self-reported mental effort interacts with the relationships, therefore this should also continue to be explored (see also Ansari \& Derakshan, 2011).

\section{Conclusions}

The present research represents an important step forward in the assessment of the impacts of anxiety on attention and visual working memory. Consistent with ACT (Eysenck et al., 2007), trait anxiety is related to efficient visual working memory performance. This was observed in increased efficiency for moderate anxiety scorers in Study 1, and decreased efficiency with higher anxiety in Study 2. In that study, the relationship between trait anxiety and performance effectiveness was also observed exclusively for the most demanding binding memory condition, and specifically trait somatic anxiety and situational stress were separate predictors. This suggests that cognitive deficits with anxiety are not necessarily underpinned by the cognitive dimension of anx, at least at the trait level, but that more demanding forms of memory may be most sensitive to trait anxiety, at least regarding performance effectiveness. Altogether, this research has demonstrated reliable relationships amongst task demand, different dimensions and levels of anxiety, situational stress, and visual working memory efficiency and effectiveness. It is hoped that these findings will help to stimulate further research within the study of anxiety and visual working memory performance. 


\section{Acknowledgements}

This research was carried out while CE, AG, LH, MM, and CM, were students at the University of Strathclyde. We thank Anna McLaughlin and Colleen Watson who also contributed to some of the data collection. Correspondence may be addressed to Louise Nicholls (School of Psychological Sciences \& Health, University of Strathclyde, 40 George Street, Glasgow, G1 1QE, UK; 1.nicholls@strath.ac.uk).

\section{References}

Allen, R. J., Atkinson, A., \& Nicholls, L. A. B., (2020). Strategic prioritisation enhances young and older adults' visual feature binding in working memory. Manuscript under review.

Allen, R. J., Baddeley, A. D., \& Hitch, G. J. (2006). Is the binding of visual features in working memory resource-demanding? Journal of Experimental Psychology: General, 135, 298-313.

Allen, R. J., Baddeley, A. D., \& Hitch, G. J. (2014). Evidence for two attentional components in visual working memory. Journal of Experimental Psychology: Learning, Memory, \& Cognition, 40, 1499-1509.

Allen, R. J., Baddeley, A. D., \& Hitch, G. J. (2017). Executive and perceptual distraction in visual working memory. Journal of Experimental Psychology: Human Perception and Performance 43, 1677-1693.

Allen, R. J., Hitch, G. J., Mate, J., \& Baddeley, A. D. (2012). Feature binding and attention in working memory: A resolution of previous contradictory findings. The Quarterly Journal of Experimental Psychology, 65, 2369-2383. 
Ansari, A. L., \& Derakshan, N. (2011). The neural correlates of cognitive effort in anxiety: Effects on processing efficiency. Biological Psychology, 86, 337-348.

Baddeley, A. D. (2007). Working memory, Thought and Action. Oxford, UK: Oxford University Press.

Baddeley, A. D. (2012). Working memory: Theories, models, and controversies. Annual Review of Psychology, 63, 1-29.

Baddeley, A. D., Allen, R. J., \& Hitch, G. J. (2011). Binding in visual working memory: The role of the episodic buffer. Neuropsychologia, 49, 1393-1400.

Baddeley, A., Banse, R., Huang, Y-M., \& Page, M. (2012). Working memory and emotion: Detecting the hedonic detector. Journal of Cognitive Psychology, 24, 6-16.

Berggren, N., Curtis, H. M., \& Derakshan, N. (2017). Interactions of emotion and anxiety on visual working memory performance. Psychonomic Bulletin \& Review, 24(4), 1274-1281.

Berggren, N., \& Derakshan, N. (2013). Attentional control deficits in trait anxiety: why you see them and why you don't. Biological Psychology, 92, 440-446.

Berggren, N., Blonievsky, T., \& Derakshan, N. (2015). Enhanced visual detection in trait anxiety. Emotion, 15, 477-483.

Berryman, C., Stanton, T. R., Bowering, K. J., Tabor, A., McFarlane, A., \& Moseley, G. L. (2013). Evidence for working memory deficits in chronic pain: a systematic review and metaanalysis. Pain, 154(8), 1181-1196. 
Berryman, C., Stanton, T. R., Bowering, K. J., Tabor, A., McFarlane, A., \& Moseley, G. L. (2014). Do people with chronic pain have impaired executive function? A meta-analytical review. Clinical Psychology Review, 34(7), 563-579.

Bishop, S. J., (2007). Neurocognitive mechanisms of anxiety: an integrative account. Trends in Cognitive Sciences, 11, 307-316.

Bleckley, M. K., Foster, J. L., \& Engle, R. W. (2015). Working memory capacity accounts for the ability to switch between object-based and location-based allocation of visual attention. Memory \& Cognition, 43, 379-388.

Brown, L. A., \& Brockmole, J. R. (2010). The role of attention in binding visual features in working memory: Evidence from cognitive ageing. The Quarterly Journal of Experimental Psychology, 63, 2067-2079.

Brown, L. A., Niven, E. H., Logie, R. H., Rhodes, S., \& Allen, R. J. (2017). Visual feature binding in younger and older adults: encoding and suffix interference effects. Memory, 25, 261-275.

Calvo, M. G. (1996). Phonological working memory and reading in test anxiety. Memory, 4(3), 289-306.

Christopher, G., \& MacDonald, J. (2005) The impact of clinical depression on working memory. Cognitive Neuropsychiatry, 10, 379-399.

Chun, M. M. (2011). Visual working memory as visual attention sustained internally over time. Neuropsychologia, 49, 1407-1409. 
Chun, M. M., Golomb, J. D., \& Turk-Browne, N. B. (2011). A taxonomy of external and internal attention. Annual Review of Psychology, 62, 73-101.

Cowan, N. (2010). The magical mystery four: how is working memory capacity limited, and why? Current Directions in Psychological Science, 19, 51-57.

Dawson, J. F. (2014). Moderation in management research: What, why, when, and how. Journal of Business and Psychology, 29, 1-19.

Edwards, E. J., Edwards, M. S., \& Lyvers, M. (2015). Cognitive trait anxiety, situational stress, and mental effort predict shifting efficiency: Implications for attentional control theory. Emotion, 15, 350-359.

Edwards, E. J., Edwards, M. S., \& Lyvers, M. (2016). Interrelationships between trait anxiety, situational stress and mental effort predict phonological processing efficiency, but not effectiveness. Emotion, 16(5), 634-646.

Edwards, M. S., Edwards, E. J., \& Lyvers, M. (2017). Cognitive trait anxiety, stress and effort interact to predict inhibitory control. Cognition and Emotion, 31, 671-686.

Egloff, B., \& Hock, M. (2001). Interactive effects of state anxiety and trait anxiety on emotional Stroop interference. Personality and Individual Differences, 31(6), 875-882.

Eysenck, M. W., Derakshan, N., Santos, R., \& Calvo, M. G. (2007). Anxiety and cognitive performance: attentional control theory. Emotion, 7, 336-353.

Eysenck, M. W., \& Derakshan, N. (2011). New perspectives in attentional control theory. Personality and Individual Differences, 50, 955-960. 
Eysenck, M., Payne, S., \& Derakshan, N. (2005) Trait anxiety, visuospatial processing, and working memory. Cognition and Emotion, 19, 1214-1228.

Figueira, J. S., Oliveira, L., Pereira, M. G., Pacheco, L. B., Lobo, I., Motta-Ribeiro, G. C., \& David, I. A. (2017). An unpleasant emotional state reduces working memory capacity: electrophysiological evidence. Social Cognitive and Affective Neuroscience, 12(6), 984-992.

Fougnie, D., \& Marois, R. (2009). Attentive tracking disrupts feature binding in visual working memory. Visual Cognition, 17, 48-66.

Gorgoraptis, N., Catalao, R. F. G., Bays, P. M., \& Husain, M. (2011). Dynamic updating of working memory resources for visual objects. The Journal of Neuroscience, 31, 8502-8511.

Grös, D. F., Antony, M. M., Simms, L. J., \& McCabe, R. E. (2007). Psychometric properties of the State-Trait Inventory for Cognitive and Somatic Anxiety (STICSA): Comparison to the State-Trait Anxiety Inventory (STAI). Psychological Assessment, 19, 369-381.

Hayes, A. F. (2017). Introduction to mediation, moderation, and conditional process analysis: A regression-based approach ( $2^{\text {nd }}$ ed.). Guilford publications.

Hitch, G. J., Allen, R. J. \& Baddeley, A. D. (2020). Attention and binding in visual working memory: Two forms of attention and two kinds of buffer storage. Attention, Perception, \& Psychophysics, 82, 280-293.

Hoffman, L., \& Rovine, M. J. (2007). Multilevel models for the experimental psychologist: Foundations and illustrative examples. Behavior Research Methods, 39, 101-117. 
Hu, Y. M., Allen, R. J., Baddeley, A. D., \& Hitch, G. J. (2016). Executive control of stimulusdriven and goal-directed attention in visual working memory. Attention Perception \& Psychophysics, 78, 2164-2175.

Hu, Y., Hitch, G. J., Baddeley, A. D., Zhang, M., \& Allen, R. J. (2014). Executive and perceptual attention play different roles in visual working memory: evidence from suffix and strategy effects. Journal of Experimental Psychology: Human Perception and Performance, 40, 1665-1678.

Jaiswal, S., Tsai, S. Y., Juan, C. H., Liang, W. K., \& Muggleton, N. G. (2018). Better cognitive performance is associated with the combination of high trait mindfulness and low trait anxiety. Frontiers in Psychology, 9, 627.

Joyce, E., Blumenthal, S., \& Wessely, S. (1996). Memory, attention, and executive function in chronic fatigue syndrome. Journal of Neurology, Neurosurgery \& Psychiatry, 60(5), 495503.

Logie, R. H. (2011). The functional organization and capacity limits of working memory. Current Directions in Psychological Science, 20, 240-245.

Luck, S. J. \& Vogel, E. K. (1997) The capacity of visual working memory for features and conjunctions. Nature, 390, 279-281.

Luck, S. J., \& Vogel, E. K. (2013). Visual working memory capacity: from psychophysics and neurobiology to individual differences. Trends in Cognitive Sciences, 17, 391-400.

Ma, W. J., Husain, M., \& Bays, P. M. (2014). Changing concepts of working memory. Nature Neuroscience, 17, 347-356. 
Markham, R., \& Darke, S. (1991). The effects of anxiety on verbal and spatial task performance. Australian Journal of Psychology, 43(2), 107-111.

Marshall, P. S., Forstot, M., Callies, A., Peterson, P. K., \& Schenck, C. H. (1997). Cognitive slowing and working memory difficulties in chronic fatigue syndrome. Psychosomatic Medicine, 59(1), 58-66.

Mella, N., Vallet, F., Beaudoin, M., Fagot, D., Baeriswyl, M., Ballhausen, N., ... Oris, M. (2018). Distinct effects of cognitive versus somatic anxiety on cognitive performance in old age: the role of working memory capacity. Aging \& Mental Health, 1-7.

Mogg, K., Mathews, A., Bird, C., \& Macgregor-Morris, R. (1990). Effects of stress and anxiety on the processing of threat stimuli. Journal of Personality and Social Psychology, 59(6), 1230-1237.

Moran, T. P. (2016). Anxiety and working memory capacity: A meta-analysis and narrative review. Psychological Bulletin, 142, 831-864.

Moreno, A. L., Ávila-Souza, J., Gomes, W. B., \& Gauer, G. (2015). Effects of worry on verbal and visual working memory. Psychology \& Neuroscience, 8, 341-349.

Moriya, J., \& Sugiura, Y. (2012). High visual working memory capacity in trait social anxiety. PLoS One, 7(4).

Moser, J. S., Becker, M. W., \& Moran, T. P. (2012). Enhanced attentional capture in trait anxiety. Emotion, 12, 213-216. 
Pacheco-Unguetti, A. P., Acosta, A., Callejas, A., \& Lupiáñez, J. (2010). Attention and anxiety: different attentional functioning under state and trait anxiety. Psychological Science, 21, 298-304.

Qi, S., Chen, J., Hitchman, G., Zeng, Q., Ding, C., Li, H., \& Hu, W. (2014). Reduced representations capacity in visual working memory in trait anxiety. Biological Psychology, $103,92-99$.

Qi, S., Ding, C., \& Li, H. (2014). Neural correlates of inefficient filtering of emotionally neutral distractors from working memory in trait anxiety. Cognitive, Affective, \& Behavioral Neuroscience, 14(1), 253-265.

Ree, M. J., French, D., MacLeod, C., \& Locke, V. (2008). Distinguishing cognitive and somatic dimensions of state and trait anxiety: Development and validation of the State-Trait Inventory for Cognitive and Somatic Anxiety (STICSA). Behavioural and Cognitive Psychotherapy, 36, 313-332.

Richards, A., French, C. C., Johnson, W., Naparstek, J., \& Williams, J. (1992). Effects of mood manipulation and anxiety on performance of an emotional Stroop task. British Journal of Psychology, 83(4), 479-491.

Sadeh, N., \& Bredemeier, K. (2011). Individual differences at high perceptual load: The relation between trait anxiety and selective attention. Cognition and Emotion, 25, 747-755.

Sari, B. A., Koster, E. H., \& Derakshan, N. (2017). The effects of active worrying on working memory capacity. Cognition and Emotion, 31, 995-1003. 
Shackman, A. J., Sarinopoulos, I., Maxwell, J. S., Pizzagalli, D. A., Lavric, A., \& Davidson, R. J. (2006). Anxiety selectively disrupts visuospatial working memory. Emotion, 6, 40-61.

Spachtholz, P., \& Kuhbandner, C. (2017). Visual long-term memory is not unitary: flexible storage of visual information as features or objects as a function of affect. Cognitive, Affective, \& Behavioral Neuroscience, 17, 1141-1150.

Spachtholz, P., Kuhbandner, C., \& Pekrun, R. (2016). Affect influences feature binding in memory: Trading between richness and strength of memory representations. Emotion, 16, 1067-1073.

Spielberger, C. D., Gorsuch, R. L, Lushene, R., Vagg, P. R., \& Jacobs, G. A. (1983). Manual for the State-trait Anxiety Inventory. Palo Alto, CA: Consulting Psychologists Press.

Vytal, K. E., Cornwell, B. R., Letkiewicz, A. M., Arkin, N. E., \& Grillon, C. (2013). The complex interaction between anxiety and cognition: insight from spatial and verbal working memory. Frontiers in Human Neuroscience, 7, 93.

Wheeler, M. E., \& Treisman, A. M. (2002). Binding in short-term visual memory. Journal of Experimental Psychology: General, 131, 48-64.

Yao, N., Chen, S., \& Qian, M. (2018). Trait anxiety is associated with a decreased visual working memory capacity for faces. Psychiatry Research, 270, 474-482. 